\title{
Influence of end-joint methods on magnetization loss in striated helical conductors
}

\author{
Woo-Seok Kima, Yungil Kim ${ }^{\text {a }}$, Kyeongdal Choi ${ }^{\text {a }}$, and Ji-Kwang Lee ${ }^{*}$ b \\ ${ }^{a}$ Korea Polytechnic University, Gyeonggi, Korea \\ ${ }^{\mathrm{b}}$ Woosuk University, Jeonbuk, Korea
}

(Received 20 November 2013; revised or reviewed 13 December 2013; accepted 14 December 2013)

\begin{abstract}
To reduce the magnetization loss of a coated conductor, the striation and the transposition have to be accomplished for magnetic decoupling. The loss reduction effect in incomplete as well as complete striated YBCO CCs was reported in previous research. At the case of the incomplete striated sample, the end region of the sample is non-striated. So, it is not jointed with each other. In power applications, the joint is needed because current leads must be connected with HTS coils. In this research, the influence of end-joint methods with copper and superconducting joint on magnetization loss in striated YBCO CC and spiral winding samples are presented and compared with non-striated measured result.
\end{abstract}

Keywords : Compact cable, magnetization loss, spiral winding, striated HTS tape, helical conductor

\section{INTRODUCTION}

The large power HTS applications require not only a large current capacity but also low AC loss characteristics of the HTS conductor as the major part of the systems. The HTS wires, which usually form a shape of thin tape, just start to be developed for large current capacity. Generally, a several number of HTS tapes should be combined to be used for large current because a single HTS wires is yet limited in current capacity so far [1]-[4]. Although many researchers are being progressed like Roebel conductors, compact cables, and HTS cable in conduit conductors for large current, none of them has shown the brightness of the usefulness for large power applications. Some of them were too expensive or too complicated to make, and others couldn't show clear reduction of AC loss. The HTS compact cable concept has been proposed by a research group of NIST and University of Colorado for large current conductor [5]. In application stage with the large current conductors, the AC loss is very important theme because it is closely related with operation efficiency. To reduce the AC loss from a bundle of the HTS single wires, we need to focus on the reduction of the magnetization loss by external magnetic field, because the magnetic field applied perpendicularly on the wide face of the HTS wire is generally dominates the $\mathrm{AC}$ loss characteristics of it. Theoretically, the magnetization loss can be reduced by making fine superconducting filaments and decoupling between them by twisting. The striation and the transposition can be adopted because it is difficult to make the filament and twist in thin plate. Striated conductor is

* Corresponding author: jikwang@woosuk.ac.kr being researched in several laboratories for low AC loss [6], [7]. It is well known that the reduction effect of AC loss disappear in incomplete striated wire due to magnetic coupling in straight conductor though the effect is in complete striated wire. But, it is unknown that helical conductor wound around the former with incomplete striated wire has the effect of AC loss reduction in external magnetic field. In this research, the measured magnetization losses of helical conductor wound with complete and incomplete striated YBCO CCs for the compact cable conductor are presented and the possibility for low loss conductor is examined. Also, in cases of complete striated CCs, the influence of end-joint type for magnetization loss is presented. Three types of end-joint including the case of incomplete striated wire are checked up and compared with the value of no jointed complete striated CC.

\section{SAMPLES AND EXPERIMENT}

The YBCO coated conductor used for samples was supplied by SuNAM Corporation in Korea and its specifications are listed in Table I. The measured critical current at self field is $153 \mathrm{~A}(77 \mathrm{~K}, 1 \mathrm{uV} / \mathrm{cm})$.

\subsection{Samples for angular dependency in helical}

We measure the magnetization loss by linked pick-up coil (LPC) method, which had been proposed by Z. Jiang and N. Amemiya [8]. First, the reliability of the measurement method using the LPC for spiral winding sample is needed. So, we prepared some HTS spiral samples with different winding pitches around a FRP 
TABLE I

SPECIFICATION OF YBCO CC

\begin{tabular}{cc}
\hline Classification & Value \\
\hline Thickness of YBCO layer & $1 \mu \mathrm{m}$ \\
Width & $4 \mathrm{~mm}$ \\
Thickness & $0.19 \mathrm{~mm}$ \\
Substrate thickness & $60 \mu \mathrm{m}$ \\
Over Layer (thickness) & $\mathrm{Ag}(2 \mu \mathrm{m})$ \\
Stabilizer & No \\
\hline
\end{tabular}

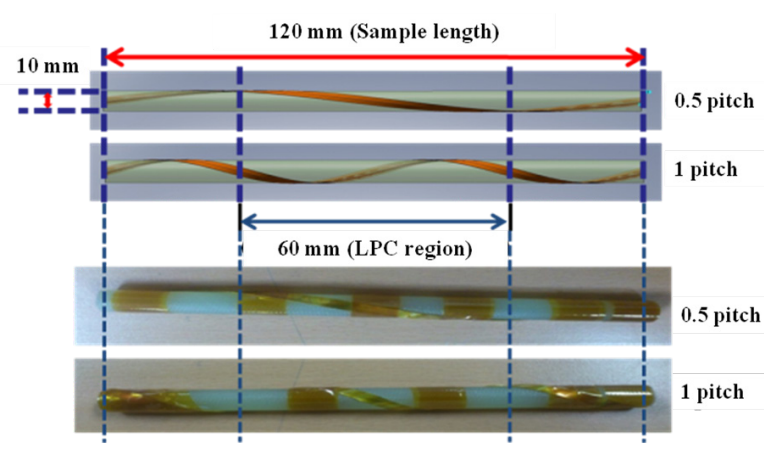

Fig. 1. Conceptual view and photographs of samples for angular dependency.

round former as shown in Fig. 1. Diameter of the former, sample length and LPC region length in spiral winding are $10 \mathrm{~mm}, 120 \mathrm{~mm}$, and $60 \mathrm{~mm}$, respectively. Winding pitches of the samples are 0.5 and 1 in LPC region. The magnetization losses of the samples were measured in external magnetic field with several incident angles. Fig. 2. is the front section of the samples in cases of the incident angles $0^{\circ}, 45^{\circ}, 90^{\circ}$ for applied external magnetic field.

\subsection{Samples for end-joint effect of magnetization loss}

Several samples with different end-joint types were prepared to investigate the loss reduction effect as shown in Table II. The samples have been helically types wound around the FRP former with striated YBCO CCs. Striated YBCO CCs are made by cutting the YBCO layer with laser. Fig. 3 is the cross-sectional view of the SEM photograph of a striated YBCO CC. The photographs and configuration of samples with several end-joint types are shown in Fig. 4.

In Table II, the character "F" means a number of filament by striation. So, the striation number is 0 (single YBCO CC, F1), 1(2-filamentary CC, F2), and 3(4-filamentary CC, F4). The sample length and the pitch length are $120 \mathrm{~mm}, 60 \mathrm{~mm}$, respectively. As shown in Fig. 4(a), the types of end-joint are incomplete, superconductor, and copper and its length is $4 \mathrm{~mm}$ in common. The superconductor and copper joint were made by soldering after cutting the YBCO layer completely (complete striation) or incompletely. The double winding spiral samples are prepared to examine the influence of winding direction as shown in Fig. 4(b). The end-joint type and jointed length is the same as for a single winding in Fig. 4(a). The character "S" and "Z" means counterclockwise and clockwise, respectively. All the samples are placed in the center of the LPC.
TABLE II

SPECIFICATION OF SAMPLES

\begin{tabular}{cc}
\hline Classification & Value \\
\hline No. of striation & No(F1), 1(F2),3(F4) \\
Jointed end length & $4 \mathrm{~mm}$ \\
Former diameter & $10 \mathrm{~mm}$ \\
Winding pitch length & $60 \mathrm{~mm}$ \\
Winding sample length & $120 \mathrm{~mm}$ \\
No. of winding & Single, Double(SS,SZ) \\
\hline
\end{tabular}

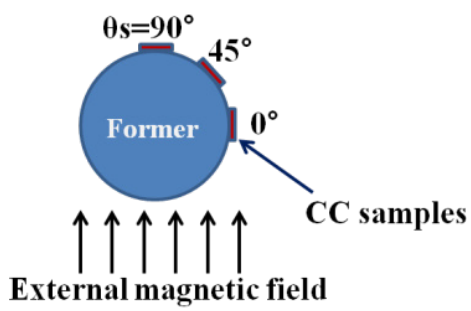

Fig. 2. Arrangement of the samples around the former (the front section).

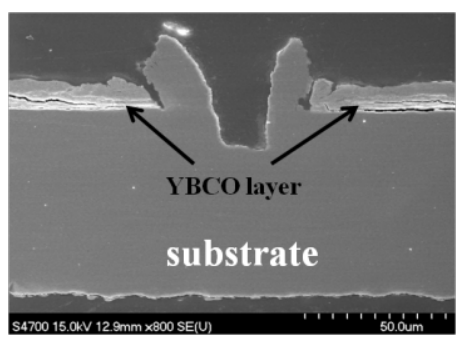

Fig. 3. Cross-sectional view of SEM photograph of a striated YBCO CC.

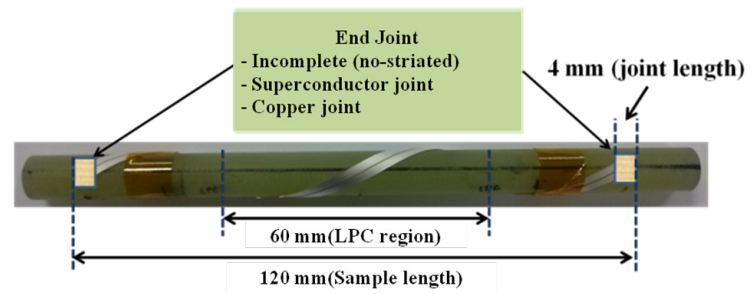

(a) End joint types of striated samples

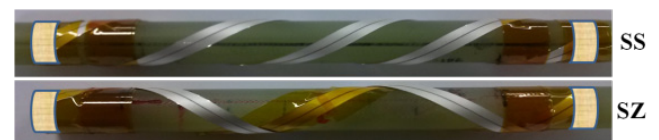

(b) Double winding samples

Fig. 4. Configuration of samples with several end-joint types.

The length of a sample in the LPC was $60 \mathrm{~mm}$ which is the same as the length of a pitch, and this region is the central part of the sample.

\subsection{Experimental set-up}

We fabricated linked pick-up coils (LPCs) to measure the loss of the HTS conductors caused by the external magnetic field. The LPCs (pick-up coil and cancel coil) 


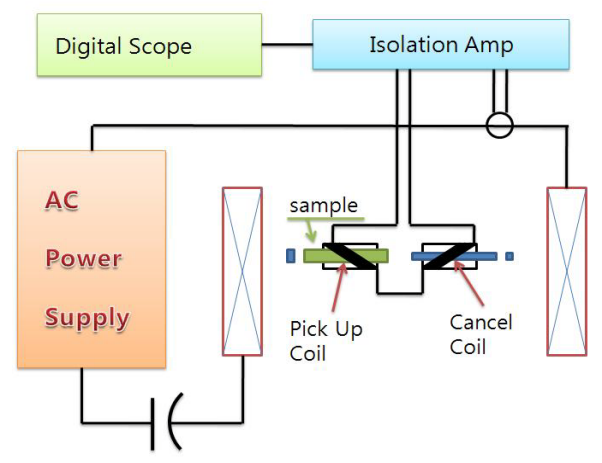

(a) Set up of magnetization loss measurement

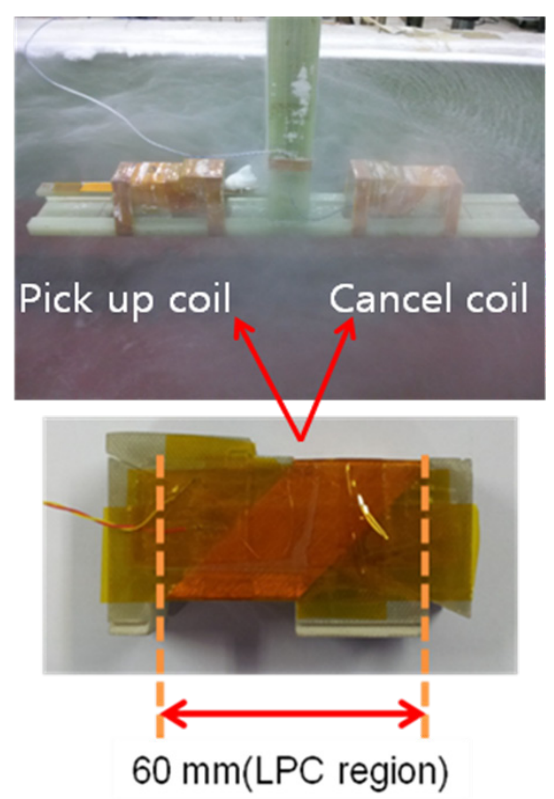

(b) Pick up and cancel coils

Fig. 5. Schematic configuration of magnetization loss mea surement system.

were wound by copper wire with diameter of $50 \mu \mathrm{m}$, and the number of turns was 600 turns each. The dimension of a single turn of the LPCs was $30 \mathrm{~mm} \times 30 \mathrm{~mm} \times 30 \mathrm{~mm}$. We also fabricated a racetrack AC magnet with uniformity within $1.0 \%$ of magnetic field in the region of LPCs to apply the uniform alternative magnetic field on the sample. Fig. 5 shows a schematic configuration of magnetization loss measurement system. The sample was placed in the center of the pick-up coil. The magnetization loss was measured by detecting the voltage (induced electric field) from the pick-up coil connected with cancel coil in anti-series and the current (applied external magnetic field) from feeding line to $\mathrm{AC}$ magnet as shown in Fig. 5.

\section{RESULTS AND DISCUSSION}

The loss density per cycle per volume is used as a unit of the loss and the frequency of applied external magnetic field is $60 \mathrm{~Hz}$ in this paper.

\subsection{Results of angular dependency in helical}

Using the LPC method, we measured the magnetization losses of the samples of Fig. 1 in different incident angles as shown in Fig. 2. The measured results are shown in Fig. 6 and Fig. 7. The losses of the samples with 0.5 pitch in the LPC region have some disagreements on the incident angles of the external magnetic field as shown in Fig. 6. However, the samples with 1.0 pitch don't show angular dependency of the external magnetic field as shown in Fig. 7. This means the magnetization loss of helical conductor has to be measured in integer pitch in LPC region for exact measurement. Therefore, all of the helical samples are with 1.0 pitch in LPC region in this paper.

\subsection{Samples for end-joint effect of magnetization loss}

To examine the loss reduction effect in helical conductor constructed with completely striated YBCO CC, the magnetization losses were measured in several samples with different numbers of striation. Fig. 8 is the measured results, and we can see that the loss reduction effect by striation appeared clearly in helical conductor such as straight conductor. In order to investigate the influence of end-joint type in helical conductor on magnetization loss reduction, the losses were measured at samples three samples with different end joints. End-joint types were incomplete, superconductor, and copper as shown in Fig. 4(a). The measured losses of the samples made by 2 and 4 filament YBCO CCs are shown in Fig. 9 and Fig. 10, respectively. The values of complete striated F2 and F4 in Fig. 8 are shown with end-jointed samples.

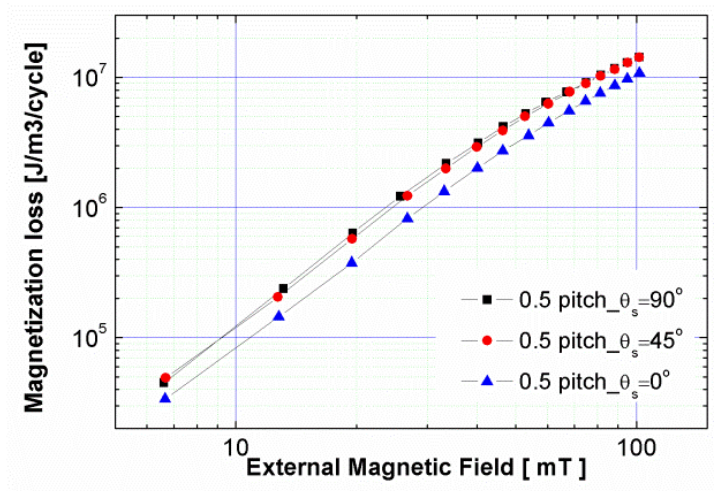

Fig. 6. The measured losses of helical samples in different incident angles which the winding pitch in LPC region is 0.5 .

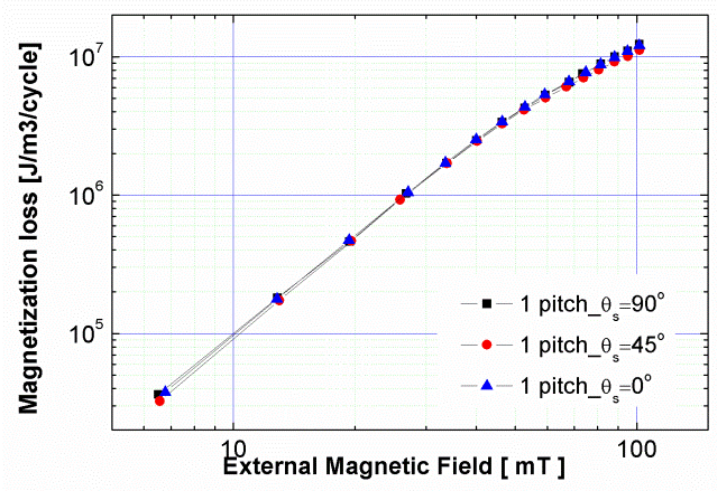

Fig. 7. The measured losses of helical samples in different incident angles which the winding pitch in LPC region is 1.0. 


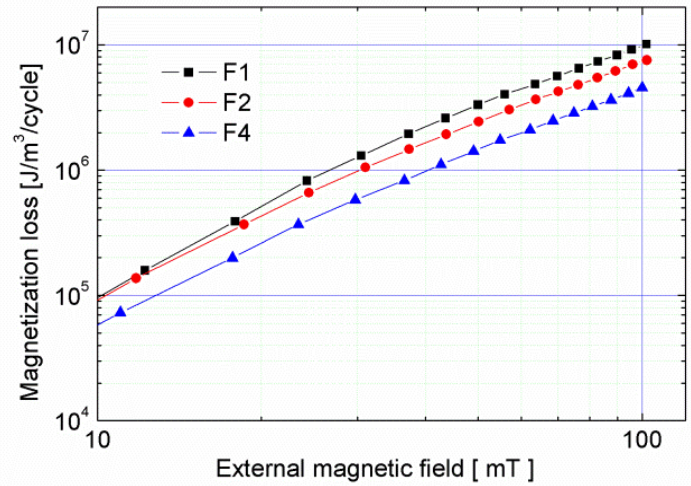

Fig. 8. The measured losses in complete striation samples with different numbers of striation.

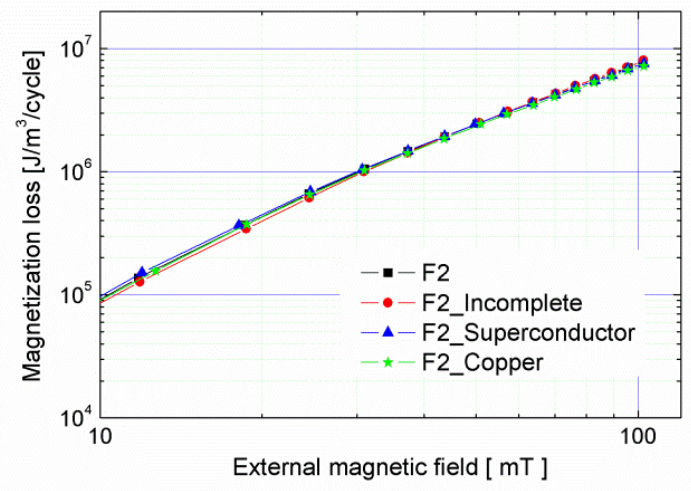

Fig. 9. The measured losses of the samples with different end-joint types, which made by 2 filament YBCO CCs.

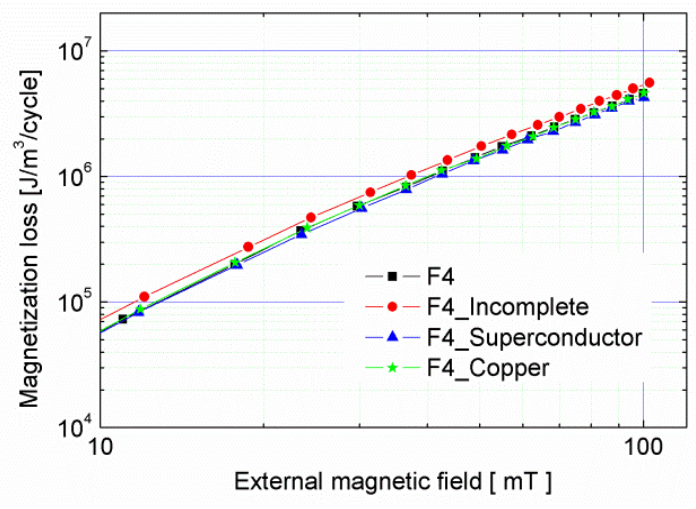

Fig. 10. The measured losses of the samples with different end-joint types, which made by 4 filament YBCO CCs.

The measured results are similar, and this means spiral shape has the effect of loss reduction in incomplete striation besides of complete striation. Also, we can see that the effect appears regardless of the end-joint types. Fig. 11 is the measured losses of double spiral winding samples SS as shown in Fig. 4(b). The effect of the loss reduction by complete striation is the same as that of single spiral winding of Fig. 8. Fig. 12 is the measured losses in SS samples with different types of end-joints, which were made by 4 filament YBCO CCs.

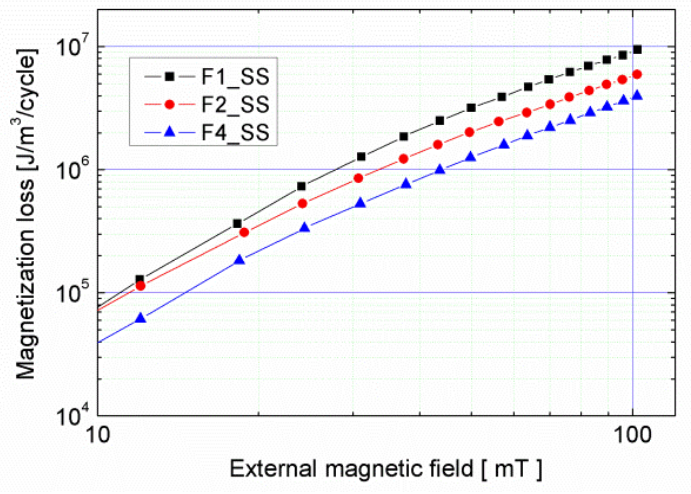

Fig. 11. The measured losses of double spiral winding samples(SS) made by complete striated YBCO CCs.

End-joint was made by a turn of band shape in end region. From the result, we can see the loss reduction effect is maintained regardless of end-joint types. Fig. 13 and Fig. 14 show the measured results in SZ samples. The values are nearly same as SS samples, and this means the winding direction is not serious in AC loss problem and the loss reduction effect is effective in any helical conductor.

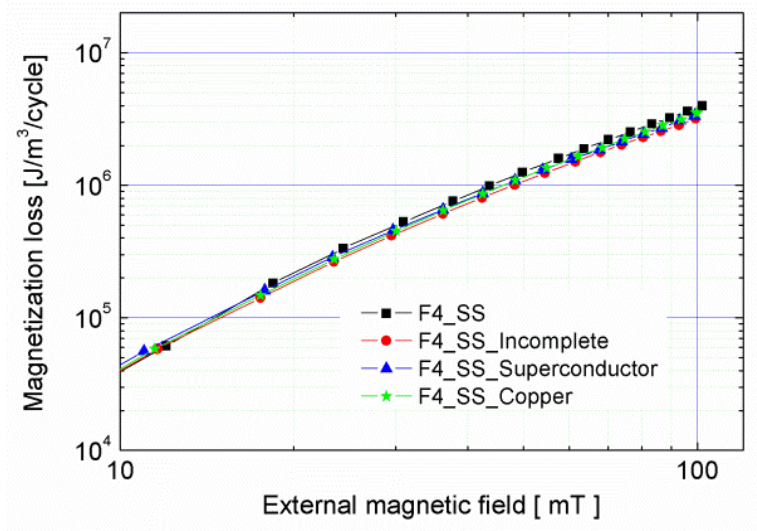

Fig. 12. The measured losses of SS samples with different end-joint types, which made by 4 filament YBCO CCs.

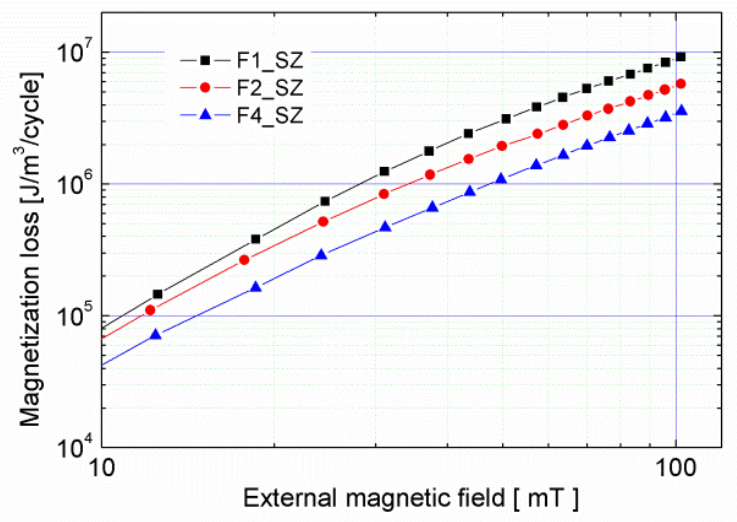

Fig. 13. The measured losses of double spiral winding samples(SZ) made by complete striated YBCO CCs. 


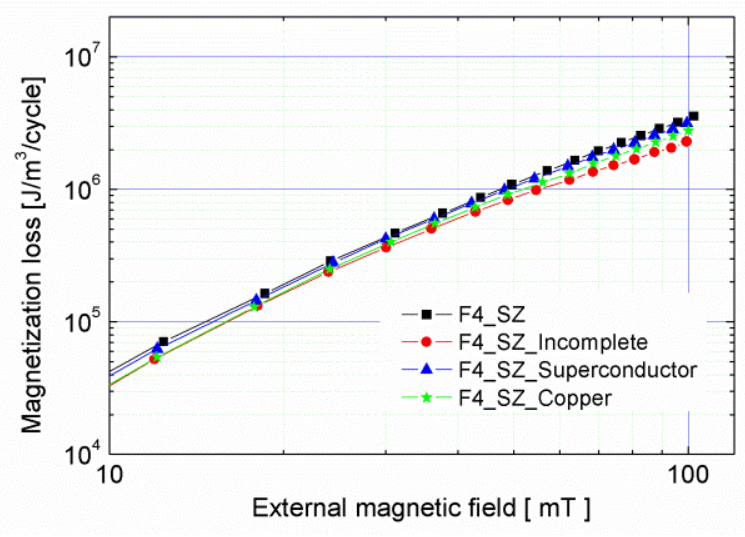

Fig. 14. The measured losses of SZ samples with different end-joint types, which made by 4 filament YBCO CCs.

\section{CONCLUSION}

It is uncertain that helical conductor wound around the former with incomplete striated wire has the effect of AC loss reduction in external magnetic field. To examine the possibility for low loss and large current capacity conductor, we measured the magnetization losses of helical conductor for the compact cable conductor. The helical conductor was constructed by winding spirally around FRP former with complete and incomplete striated YBCO CCs. To examine the influence of end-joint type for magnetization loss, three types of end-joint including the case of incomplete striated wire are checked up and compared with the value of no jointed complete striated CC. From the measured results, it is verified that striation (incomplete as well as complete) gives the effect of AC loss reduction in helical conductor regardless of the type of end-joint.

\section{ACKNOWLEDGMENT}

This research was supported by Basic Science Research Program though the National Research Foundation of Korea(NRF) funded by the Ministry of Education, Science and Technology(2012R1A1A4A01012300), Republic of Korea.

\section{REFERENCES}

[1] Myungjin Park et al., "Effect of the stack in HTS tapes exposed to external magnetic field," IEEE Trans. Applied Superconductivity, vol. 14, no. 2, pp. 1106-1109, 2004.

[2] S. Schuller., "Ac-loss measurement of a DyBCO-Roebel assembled coated conductor cable (RACC)," Physica C: Superconductivity, vol. 463-465, pp. 761-765, 2007.

[3] Jiang, Z et al., "Total AC loss characteristics in a stacked YBCO conductor," IEEE Trans. Applied Superconductivity, vol. 17, no. 2, pp. 2442-2445, 2007.

[4] W. Goldacker et al., "ROEBEL assembled coated conductors(RACC): preparation, properties and progress," IEEE Trans. Applied Superconductivity, vol. 17, no. 2, pp. 3398-3401, 2007.

[5] D C van der Laan et al., "Compact GdBa2Cu3O7-d coated conductor cables for electric power transmission and magnet application," Supercond. Sci. Technol, issue 4, 24, 2011.

[6] Ogawa, J et al., "Magnetization loss in a striated YBCO coated conductor considering the intrinsic critical current distribution," IEEE Trans. Applied Superconductivity, vol. 16, no. 2, pp. 111-114, 2006.

[7] J.K. Lee et al., "Reduction effect on magnetization loss in the stacked conductor with striated and transposed YBCO coated conductor," IEEE Trans. Applied Superconductivity, vol. 19, no. 3, pp. 3340-3343, 2009.

[8] Z. Jiang and N. Amemiya, "An experimental method for total AC loss measurement of high Tc superconductors," Supercond Sci. Technol., vol. 17, no. 3, pp. 371-379, 2004. 